hep-th/0307254

CERN-TH/2003-165

\title{
Suppressing the Cosmological Constant in Non-Supersymmetric Type I Strings
}

\author{
Carlo Angelantonj and Ignatios Antoniadis ${ }^{\dagger}$ \\ CERN - Theory Division, CH-1211 Geneva 23
}

\begin{abstract}
We construct non-supersymmetric type I string models which correspond to consistent flatspace solutions of all classical equations of motion. Moreover, the one-loop vacuum energy is naturally fixed by the size of compact extra dimensions which, in the two-dimensional case, can be lowered to a fraction of a millimetre. This class of models has interesting non-abelian gauge groups and can accommodate chiral fermions. In the large radius limit, supersymmetry is recovered in the bulk, while D-brane excitations, although nonsupersymmetric, exhibit Fermi-Bose degeneracy at all mass levels. We also give some evidence for a suppression of higher-loop corrections to the vacuum energy.
\end{abstract}

July 2003

$\dagger$ On leave from CPHT École Polytechnique (UMR du CNRS 7644), F-91128 Palaiseau 


\section{Introduction}

One of the outstanding problems in string theory, as in any quantum theory including gravity, is to understand the smallness of the cosmological constant. This problem became much more severe after the recent observations suggesting a non-vanishing value corresponding to a new energy scale, by far smaller than every other scale in physics of fundamental interactions, $\Lambda \equiv E_{\Lambda}^{4}$ with $E_{\Lambda} \sim 10^{-4} \mathrm{eV}$. On the other hand, brane-world models with large extra dimensions have recently received a lot of attention, as they offer a new framework for stabilising the mass hierarchy and addressing several problems of particle physics in an interesting new perspective [1.2]. In particular, models with two large flat dimensions of submillimeter size are particularly attractive for manifold reasons: technical (maximum transverse space available in phenomenologically interesting string constructions); phenomenological (enhanced signals both in particle colliders and in micro-gravity experiments); and theoretical, related mainly to the logarithmic growth of massless field propagation in the bulk [3, 四, offering new ways for addressing gauge coupling unification, neutrino masses and oscillations, fixing the radion, etc.

It is then suggestive, if not a simple numerical coincidence, that the size of the bulk $R_{\perp}$ in these models is of the same order of magnitude as the cosmological constant scale $R_{\perp} \sim E_{\Lambda}^{-1} \sim 1 \mathrm{~mm}^{\star}$. In fact, the behaviour $\Lambda \sim 1 / R_{\perp}^{4}$ is generically valid in models where supersymmetry is broken by Scherk-Schwarz boundary conditions [6, 0,8,9, 9, 10, 1, 11]. However, in this case the induced mass splittings are of order $1 / R_{\perp}$, and thus too small by several orders of magnitude. It would then be very appealing if supersymmetry might be broken on the branes at the string scale $M_{s} \sim 1 \mathrm{TeV}$, in a way that the vacuum energy vanishes in the decompactification limit. Note that in this limit four-dimensional gravity decouples, since the effective Planck mass $M_{P} \simeq M_{s}^{2} R_{\perp}$ goes to infinity.

Type II string models with possible vanishing cosmological constant in perturbation theory were studied in refs. [12,13]. Their main feature is a Fermi-Bose degenerate spectrum, thus leading to an automatic vanishing of the one-loop vacuum energy. Aside from the question of possible higher-loop corrections [14], their main defect, however, was that the non-abelian gauge sector, appearing at particular singular points of the compactification manifold, or on appropriate D-brane collections, was always supersymmetric [15, 16].

* See [5] where similar ideas for suppressing the cosmological constant have been exposed, though employing different methods. 
Thus, it is questionable whether such constructions can accommodate gauge degrees of freedom with large supersymmetry-breaking mass splittings.

In this work, we address this problem and construct type I string models with nonsupersymmetric D-brane spectra and an additional source of supersymmetry breaking in the bulk induced by Scherk-Schwarz boundary conditions. In the large radius limit, the one-loop cosmological constant vanishes as $1 / R_{\perp}^{4}$, while the $\mathrm{D}$-brane spectra exhibit fermion-boson degeneracy at all massive levels. In the simplest case, this degeneracy can be thought of as emerging from a supersymmetric theory, where superpartners have been displaced appropriately in the position space.

Our paper is organised as follows. In section 2, we present a brief review of the socalled M-theory breaking mechanism, exhibiting either "brane supersymmetry" or "brane supersymmetry breaking". In section 3, we describe how discrete deformations can trade

$\mathscr{O}^{+}$planes for $\mathscr{O}^{-}$ones. In section 4 , we construct the a simple model exhibiting open-string Fermi-Bose degeneracy in the large-radius limit, at all mass levels. The one-loop vacuum energy is computed in section 5, where we show the previously announced behaviour. In section 6, we generalise the construction to lower dimensions. Finally, in section 7, we comment on the possibility that higher-order corrections do not destabilise the one-loop result.

\section{M-theory breaking revisited}

In order to make our analysis more explicit, it is worth to review in this section the basic features of the "M-theory breaking" mechanism, exhibiting the phenomena of either "brane supersymmetry" [8] or "brane supersymmetry breaking" [17, 1].

In oriented closed-string theories, the Scherk-Schwarz reduction results from a discrete deformation compatible with modular invariance [7]. The general method for breaking supersymmetry by compactification uses a (discrete) R-symmetry of the higher-dimensional theory and couples the lattice momenta to the corresponding R-charges. In its simplest type IIB nine-dimensional manifestation, which is also the most suited for our purposes, the partition function reads

$$
\begin{aligned}
\mathscr{T}= & \left(V_{8} \bar{V}_{8}+S_{8} \bar{S}_{8}\right) \Gamma_{m, 2 n}^{(1,1)}+\left(O_{8} \bar{O}_{8}+C_{8} \bar{C}_{8}\right) \Gamma_{m, 2 n+1}^{(1,1)} \\
& -\left(V_{8} \bar{S}_{8}+S_{8} \bar{V}_{8}\right) \Gamma_{m+\frac{1}{2}, 2 n}^{(1,1)}-\left(O_{8} \bar{C}_{8}+C_{8} \bar{O}_{8}\right) \Gamma_{m+\frac{1}{2}, 2 n+1}^{(1,1)},
\end{aligned}
$$


with $\Gamma_{m, n}^{(1,1)}$ the $(1,1)$-dimensional Narain lattice associated to a circle of radius $R$, with Kaluza-Klein momentum $m$ and winding $n$. For notational simplicity, the summation over momenta and windings is suppressed, and we follow the notation of [18]. $O_{n}, V_{n}, S_{n}$ and $C_{n}$ are the $\mathrm{SO}(n)$ characters corresponding to the identity, the vector, the spinor and the conjugate spinor representations. In this string generalisation of the Scherk-Schwarz mechanism, all space-time fermions with even windings have evidently masses shifted by $1 / 2 R$ as compared to their would-be bosonic superpartners, while in the odd-winding sector there is also a reversed GSO projection.

The model (2.1) with supersymmetry breaking is still invariant under world-sheet parity $\Omega$ that, henceforth, can be gauged [19]. We are actually interested in T-dual IIA orientifold models obtained by an $\Omega \mathscr{I}$ projection, where $\mathscr{I}$ is the inversion of the compact coordinate $x^{9}$. The corresponding Klein-bottle amplitude reads

$$
\mathscr{K}=\frac{1}{2}\left(V_{8}-S_{8}\right) W_{2 n}^{(1)}+\frac{1}{2}\left(O_{8}-C_{8}\right) W_{2 n+1}^{(1)},
$$

and, consequently,

$$
\tilde{\mathscr{K}}=\frac{2^{5}}{2 R}\left(V_{8} P_{2 m}^{(1)}-S_{8} P_{2 m+1}^{(1)}\right),
$$

where, as usual, the tilde in the amplitude stands for its transverse-channel (i.e. closedstring) representation, and $P^{(1)}, W^{(1)}$ denote the momentum or winding partition functions: $P_{m}^{(1)} \equiv \Gamma_{m, 0}^{(1,1)}, W_{n}^{(1)} \equiv \Gamma_{0, n}^{(1,1)}$. From the expression (2.3) one can immediately read the geometry of the orientifold planes introduced by the $\Omega \mathscr{I}$ projection [20]. Indeed, after rewriting (2.3) in the form

$$
\tilde{\mathscr{K}}=\frac{2^{5}}{2 R}\left[V_{8}\left(\frac{1+(-1)^{m}}{2}\right)^{2}-S_{8}\left(\frac{1-(-1)^{m}}{2}\right)^{2}\right] P_{m}^{(1)},
$$

one can see that two orientifold 8-planes are sitting at the edges of the segment $S^{1} / \mathbb{Z}_{2}$, i.e. at the fixed points of the $\Omega \mathscr{I}$ involution, and, moreover the relative minus sign in the $S_{8}$ coefficient neatly reveals that the two planes are CPT conjugate, namely $\mathscr{O}^{+}$and $\overline{\mathscr{O}}^{+}$. Overall, one has the configuration of figure 1.

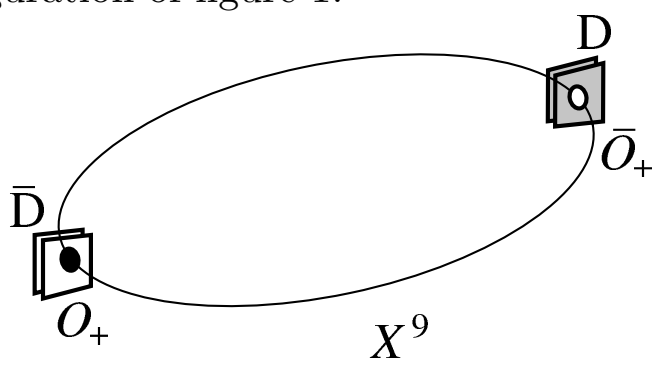

Fig.1. The geometry of the nine-dimensional M-theory breaking model 
As usual, tadpole cancellation demands the introduction of an open-string sector that, in the case at hand, is bound to involve brane-antibrane pairs. The annulus amplitude then reads

$$
\mathscr{A}=\frac{1}{2}\left(N_{\mathrm{D}}^{2}+N_{\overline{\mathrm{D}}}^{2}\right)\left(V_{8}-S_{8}\right) W_{n}^{(1)}+N_{\mathrm{D}} N_{\overline{\mathrm{D}}}\left(O_{8}-C_{8}\right) W_{n+\frac{1}{2}},
$$

where the first term describes open strings ending on a stack of $N_{\mathrm{D}}$ branes or $N_{\overline{\mathrm{D}}}$ antibranes, while the second term, with reverted GSO projection, pertains to open strings stretched between branes and antibranes. An $S$-modular transformation, brings the annulus amplitude in the transverse-channel description

$$
\begin{aligned}
\tilde{\mathscr{A}} & =\frac{2^{-5}}{2 R}\left[\left(N_{\mathrm{D}}+N_{\overline{\mathrm{D}}}\right)^{2}\left(V_{8} P_{2 m}^{(1)}-S_{8} P_{2 m+1}^{(1)}\right)+\left(N_{\mathrm{D}}-N_{\overline{\mathrm{D}}}\right)^{2}\left(V_{8} P_{2 m+1}^{(1)}-S_{8} P_{2 m}^{(1)}\right)\right] \\
& =\frac{2^{-5}}{2 R}\left[\left((-1)^{m} N_{\mathrm{D}}+N_{\overline{\mathrm{D}}}\right)^{2} V_{8}-\left((-1)^{m} N_{\mathrm{D}}-N_{\overline{\mathrm{D}}}\right)^{2} S_{8}\right] P_{m}^{(1)},
\end{aligned}
$$

that, actually, makes transparent our choice of Wilson lines, or brane displacements: we have here decided to put the $N_{\mathrm{D}}$ branes on top of the $\overline{\mathscr{O}}^{+}$plane at $x^{9}=\pi R$, while the $N_{\overline{\mathrm{D}}}$ antibranes are at $x^{9}=0$, where an $\mathscr{O}^{+}$plane lives ${ }^{\dagger}$.

Finally, the transverse-channel Möbius amplitude

$$
\tilde{\mathscr{M}}=-\frac{1}{R}\left[\left(\frac{1+(-1)^{m}}{2}\right)\left(N_{\mathrm{D}}+N_{\overline{\mathrm{D}}}\right) V_{8}+\left(\frac{1-(-1)^{m}}{2}\right)\left(N_{\mathrm{D}}+N_{\overline{\mathrm{D}}}\right) S_{8}\right] P_{m}^{(1)}
$$

is completely determined by $\tilde{\mathscr{K}}$ and $\tilde{\mathscr{A}}$, and in the direct channel

$$
\mathscr{M}=-\frac{1}{2}\left[\left(N_{\mathrm{D}}+N_{\overline{\mathrm{D}}}\right) V_{8}+\left(N_{\mathrm{D}}+N_{\overline{\mathrm{D}}}\right) S_{8}(-1)^{n}\right] W_{n}^{(1)}
$$

gives a proper (anti)symmetrisation of $\mathscr{A}$.

At the massless level one gets an $\mathrm{SO}(16) \otimes \mathrm{SO}(16)$ Chan-Paton gauge group with fermions in the $(136,1) \oplus(1,136)$ representation. In this open-string sector supersymmetry is broken directly at the string scale as a consequence of our choice of Wilson lines [4]. This breaking in the open-string sector is closer in spirit to the "brane supersymmetry breaking" mechanism of [17] rather than to the Scherk-Schwarz reduction [8]. The latter would correspond to exchange branes with antibranes, giving rise to supersymmetric massless open-string excitations (brane supersymmetry).

$\dagger$ Here we use the conventions of [21]: an $\mathscr{O}^{ \pm}$plane has both negative (positive) tension and charge, while a bar denotes its CPT conjugate with reversed R-R charge. 
The one-loop vacuum energy density results from the contributions of the four surfaces of vanishing Euler character

$$
\begin{aligned}
\Lambda(R)= & \frac{1}{2} \int_{\mathscr{F}} \frac{d^{2} \tau}{\tau_{2}^{11 / 2}} \frac{\mathscr{T}(R)}{|\eta|^{14}}+\int_{0}^{\infty} \frac{d \tau_{2}}{\tau_{2}^{11 / 2}} \frac{\mathscr{K}(R)}{\eta^{7}\left(2 i \tau_{2}\right)} \\
& +\int_{0}^{\infty} \frac{d \tau_{2}}{\tau_{2}^{11 / 2}} \frac{\mathscr{A}(R)}{\eta^{7}\left(\frac{i \tau_{2}}{2}\right)}+\int_{0}^{\infty} \frac{d \tau_{2}}{\tau_{2}^{11 / 2}} \frac{\mathscr{M}(R)}{\eta^{7}\left(\frac{1}{2}+\frac{i \tau_{2}}{2}\right)}
\end{aligned}
$$

where $\mathscr{F}$ is the familiar $\operatorname{SL}(2, \mathbb{Z})$ fundamental domain for the complex modulus $\tau$ of the world-sheet torus. From the expressions (2.1), (2.2), (2.4) and (2.5), it is then clear that $\mathscr{T}, \mathscr{K}$ and $\mathscr{A}$ give rise to a cosmological constant which is power-law or exponentially suppressed with the size of the compact Scherk-Schwarz dimension, while this is not the case for $\mathscr{M}$ that yields, instead, a sizable result set by the string scale. This is a direct consequence of the miss-matching between bosonic and fermionic degrees of freedom on the branes. Actually, in the UV regime $\tau_{2} \rightarrow 0, \mathscr{K}, \mathscr{A}$ and $\mathscr{M}$, independently, develop linear in $R$ contributions, related to the emergence of local tadpoles. Indeed, the general large-radius limit of the one-loop cosmological constant in four dimensions is [4,22]

$$
\Lambda(R) \sim\left(n_{\mathrm{B}}^{c}-n_{\mathrm{F}}^{c}\right) \frac{1}{R^{4}}+c_{1} M_{s}^{4}+c_{2}\left(n_{\mathrm{B}}^{o}-n_{\mathrm{F}}^{o}\right) \frac{M_{s}^{6-n}}{R^{n-2}}+\mathscr{O}\left(e^{-M_{s}^{2} R^{2}}\right)
$$

where $n_{\mathrm{B}, \mathrm{F}}^{c}$ and $n_{\mathrm{B}, \mathrm{F}}^{o}$ denote the number of massless bosonic and fermionic degrees of freedom from the closed and open string sectors, respectively. The first term is a consequence of spontaneous supersymmetry breaking by Scherk-Schwarz boundary conditions in the closed-string sector [10,11,11, while the second term is expected from the absence of supersymmetry on the branes, leading to a non-vanishing localised energy density of order of the string scale. Finally, the third term is a consequence of non-vanishing local tadpoles of massless closed-string states, and its large radius behaviour depends on the number $n$ of dimensions transverse to the branes that have a size comparable to $R$ [4, 22] .

Can we improve our model, associating to the closed-string spectrum of (2.1) and (2.2) an open-string sector still non-supersymmetric but with Fermi-Bose degeneracy, at least at the massless level? In other words, can we trade the $\overline{\mathscr{O}}^{+}$plane at $x^{9}=\pi R$ for an $\overline{\mathscr{O}}^{-}$one, in order to symmetrise the gauge bosons living on the D-branes, while still anti-symmetrising the associated fermions? 


\section{Introducing discrete deformations}

In a given model, the nature of the $\mathscr{O}$ planes content does not depend solely on the type of the orientifold group $\mathscr{G}_{\Omega}$, but also on the values of the background fields. Indeed, a simple way to transmute $\mathscr{O}$ planes consists in introducing discrete deformations in the closed-string sector [21]. Although $\mathscr{G}_{\Omega}$ projects out some of the excitations of the parent theory, it was shown originally in [23] that world-sheet parity is still compatible with quantised values for their backgrounds. The simplest instance where this phenomenon occurs is the compactification on a two-dimensional torus with generic complex and Kähler structures. The left-handed and right-handed momenta are then

$$
\begin{aligned}
& p_{\mathrm{L}, a}=m_{a}+\frac{1}{\alpha^{\prime}}\left(g_{a b}-B_{a b}\right) n^{b}, \\
& p_{\mathrm{R}, a}=m_{a}-\frac{1}{\alpha^{\prime}}\left(g_{a b}+B_{a b}\right) n^{b} .
\end{aligned}
$$

Although asymmetric for generic values of the metric $g_{a b}$ and the two-index antisymmetric tensor $B_{a b}$, the Narain lattice can be made compatible with world-sheet parity if the NS-NS two-form is quantised 23

$$
B_{a b}=\frac{\alpha^{\prime}}{2} k \epsilon_{a b}
$$

with $\epsilon_{a b}$ the Levi-Civita two-tensor and $k$ an integer. Combined with the Peccei-Quinn translations, eq. (3.1) then identifies two inequivalent classes:

$$
B_{a b}=0, \quad B_{a b}=\frac{\alpha^{\prime}}{2} \epsilon_{a b}
$$

In orientifold constructions, the first class corresponds to standard compactifications (with vector structure) involving, in the T-dual version, four identical $\mathscr{O}^{+}$planes sitting at the four fixed points of $T^{2} / \mathbb{Z}_{2}$. The second class, on the other hand, is associated to $T^{2}$ 's without vector structure, and has the effect of halving the net charge and tension of the $\mathscr{O}$ planes involved [21]. Indeed, the associated transverse-channel Klein-bottle amplitude (in the case of an orthogonal torus with radii $R_{1}$ and $R_{2}$ )

$$
\begin{aligned}
\tilde{\mathscr{K}}= & \frac{2^{3}}{2} \frac{\alpha^{\prime}}{R_{1} R_{2}}\left(V_{8}-S_{8}\right) P_{\left(m_{1}, m_{2}\right)} \\
& =\frac{2^{3}}{2} \frac{\alpha^{\prime}}{R_{1} R_{2}}\left(V_{8}-S_{8}\right)\left(\frac{1-(-1)^{m_{1}}+(-1)^{m_{2}}+(-1)^{m_{1}+m_{2}}}{2}\right)^{2} P_{\left(m_{1}, m_{2}\right)}
\end{aligned}
$$

clearly shows the origin of this reduction: among the four orientifold planes, three have both negative tension and charge, i.e. they are the familiar $\mathscr{O}^{+}$planes, while the fourth 
one, at say $\left(x^{1}, x^{2}\right)=\left(\pi R_{1}, 0\right)$, has reversed tension and charge, and is usually denoted as $\mathscr{O}^{-}$plane (see fig. 2).
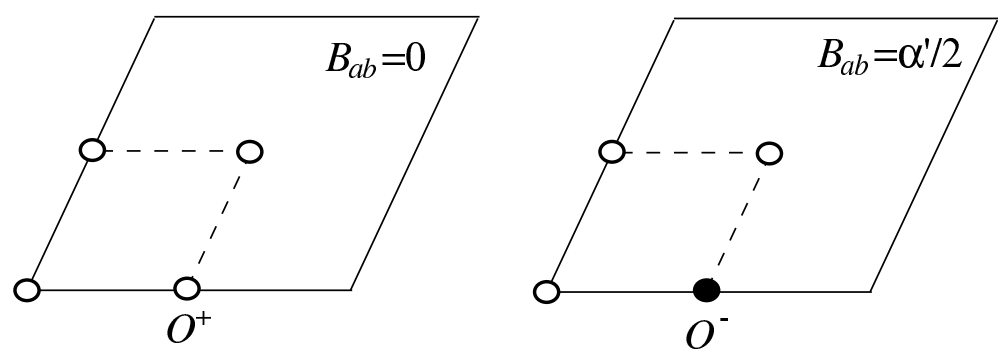

Fig. 2. The $\mathscr{O}$ plane configuration for a non-trivial $B_{a b}$ background

As a result, tadpole conditions now call for the introduction of only sixteen D-branes (or eight $\mathscr{G}_{\Omega}$ invariant combinations, according to different ways of counting) and the associated gauge group is orthogonal or symplectic depending on the nature of the orientifold plane they are next to $\left(\mathscr{O}^{+}\right.$or $\mathscr{O}^{-}$, respectively) [23].

\section{The model}

We have now got all the ingredients to concoct the model we are looking for. One has simply to compactify the nine-dimensional model we described in section 2 on an additional $T^{2}$ without vector structure, i.e. with a non-vanishing $B$-field, and distribute the D-branes as in figure 3.

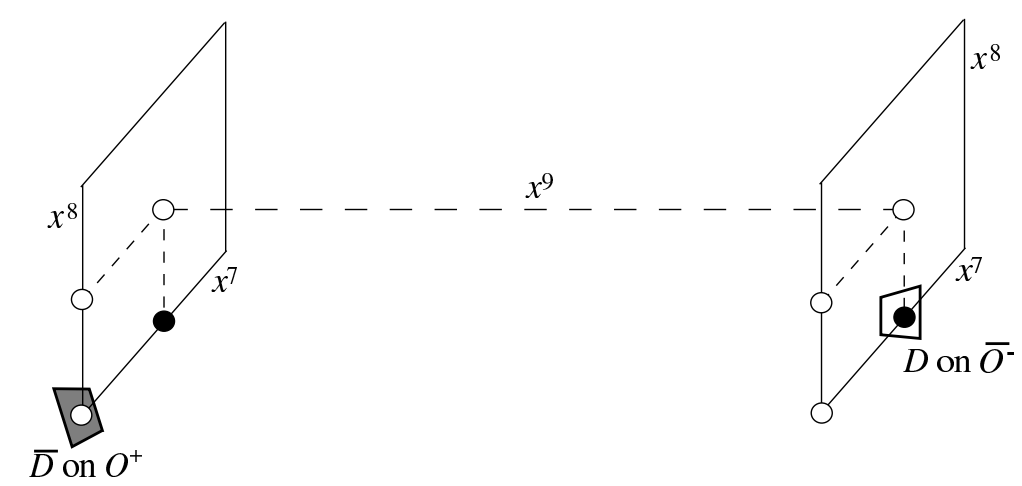

Fig. 3. O-planes and D-branes configurations for the seven-dimensional orientifold. 
To be more concrete, we start from the type IIB model (2.1) compactified on a $(2,2)$ dimensional lattice, $\Gamma^{(2,2)}(B)$, with the NS-NS two-form $B_{a b}=\frac{\alpha^{\prime}}{2} \epsilon_{a b}$,

$$
\begin{aligned}
\mathscr{T}= & {\left[\left(V_{8} \bar{V}_{8}+S_{8} \bar{S}_{8}\right) \Gamma_{m, 2 n}^{(1,1)}+\left(O_{8} \bar{O}_{8}+C_{8} \bar{C}_{8}\right) \Gamma_{m, 2 n+1}^{(1,1)}\right.} \\
& \left.-\left(V_{8} \bar{S}_{8}+S_{8} \bar{V}_{8}\right) \Gamma_{m+\frac{1}{2}, 2 n}^{(1,1)}-\left(O_{8} \bar{C}_{8}+C_{8} \bar{O}_{8}\right) \Gamma_{m+\frac{1}{2}, 2 n+1}^{(1,1)}\right] \Gamma^{(2,2)}(B) .
\end{aligned}
$$

To fix the notation, $x^{9}$ is the coordinate on the circle of radius $R$ responsible for the ScherkSchwarz deformation, with $m$ and $n$ its associated momenta and windings. Coordinates on the spectator $T^{2}$ are labelled by the pair $\left(x^{7}, x^{8}\right)$. For simplicity we choose $T^{2}$ to be a squared torus with sides of size $2 \pi R_{7}$ and $2 \pi R_{8}$, and, of course, with a background $B_{a b}=\frac{\alpha^{\prime}}{2} \epsilon_{a b}$. The corresponding momenta and windings are labelled by $\left(m_{7}, m_{8}\right)$ and $\left(n^{7}, n^{8}\right)$.

Thus, we can immediately write down the Klein-bottle amplitude

$$
\mathscr{K}=\frac{1}{2}\left[\left(V_{8}-S_{8}\right) W_{2 n}^{(1)}+\left(O_{8}-C_{8}\right) W_{2 n+1}^{(1)}\right] W_{\left(2 n^{7}, 2 n^{8}\right)}^{(2)}
$$

associated to the $\Omega \mathscr{I}$ orientifold projection, with now $\mathscr{I} x^{i}=-x^{i}$, an inversion on the three compact coordinates, $i=7,8,9$.

The massless closed unoriented spectrum is simply given by the dimensional reduction of that in section 2. In terms of ten-dimensional fields it comprises the graviton, the dilaton and a $\mathrm{R}-\mathrm{R}$ two-form potential. For $R<\sqrt{\alpha^{\prime}}$ a tachyon appears in the spectrum in the odd-winding (twisted) sector. However, in this paper we are only interested in very large values for $R$ and thus we are not concerned with this.

An $S$ modular transformation maps $\mathscr{K}$ into the transverse-channel amplitude

$$
\begin{aligned}
\tilde{\mathscr{K}}= & \frac{2^{5}}{2 \cdot 4} \frac{\left(\alpha^{\prime}\right)^{3 / 2}}{R R_{8} R_{7}}\left[V_{8}\left(\frac{1+(-1)^{m}}{2}\right)^{2}-S_{8}\left(\frac{1-(-1)^{m}}{2}\right)^{2}\right] P_{m}^{(1)} \\
& \times\left(\frac{1-(-1)^{m_{7}}+(-1)^{m_{8}}+(-1)^{m_{7}+m_{8}}}{2}\right)^{2} P_{\left(m_{7}, m_{8}\right)}^{(2)} .
\end{aligned}
$$

As expected, two CPT-conjugate copies of the r.h.s two-torus of figure 2 are sitting at the edges of the $x^{9}$ segment, as shown in figure 3 .

The rest of the construction is now straightforward. We have simply to add eight brane-antibrane pairs to cancel the NS-NS tadpole in $\tilde{\mathscr{K}}$. As usual, in the open sector we 
have the freedom to distribute the branes along the compact dimensions. In the case at hand, the configuration of figure 3 corresponds to the annulus amplitude

$$
\mathscr{A}=\frac{1}{2}\left(N_{\mathrm{D}}^{2}+N_{\overline{\mathrm{D}}}^{2}\right)\left(V_{8}-S_{8}\right) W_{n}^{(1)} W_{\left(n^{7}, n^{8}\right)}^{(2)}+N_{\mathrm{D}} N_{\overline{\mathrm{D}}}\left(O_{8}-C_{8}\right) W_{n+\frac{1}{2}}^{(1)} W_{\left(n^{7}+\frac{1}{2}, n^{8}\right)}^{(2)},
$$

where, as before, $N_{\mathrm{D}}\left(N_{\overline{\mathrm{D}}}\right)$ counts the number of branes (antibranes).

From

$$
\begin{aligned}
\tilde{\mathscr{A}}= & \frac{2^{-5}}{2} \frac{\left(\alpha^{\prime}\right)^{3 / 2}}{R R_{8} R_{7}}\left[\left((-1)^{m}(-1)^{m_{7}} N_{\mathrm{D}}+N_{\overline{\mathrm{D}}}\right)^{2} V_{8}\right. \\
& \left.-\left((-1)^{m}(-1)^{m_{7}} N_{\mathrm{D}}-N_{\overline{\mathrm{D}}}\right)^{2} S_{8}\right] P_{m}^{(1)} P_{\left(m_{7}, m_{8}\right)}^{(2)}
\end{aligned}
$$

and $\tilde{\mathscr{K}}$ we can immediately extract the Möbius amplitude in the transverse channel

$$
\begin{aligned}
\tilde{\mathscr{M}}= & -\frac{1}{2} \frac{\left(\alpha^{\prime}\right)^{3 / 2}}{R R_{8} R_{7}}\left[V_{8}\left(\frac{1+(-1)^{m}}{2}\right)+S_{8}\left(\frac{1-(-1)^{m}}{2}\right)\right] P_{m}^{(1)} \\
& \times\left(\frac{1-(-1)^{m_{7}}+(-1)^{m_{8}}+(-1)^{m_{7}+m_{8}}}{2}\right)\left((-1)^{m_{7}} N_{\mathrm{D}}+N_{\overline{\mathrm{D}}}\right) P_{\left(m_{7}, m_{8}\right)}^{(2)} .
\end{aligned}
$$

Upon a $P$-modular transformation, one then finds

$$
\mathscr{M}=-\frac{1}{2}\left(V_{8}+(-1)^{n} S_{8}\right) W_{n}^{(1)}\left[\left(N_{\mathrm{D}}+N_{\overline{\mathrm{D}}}\right) W_{\left(n^{7}, 2 n^{8}+1\right)}^{(2)}-\left(N_{\mathrm{D}}-N_{\overline{\mathrm{D}}}\right)(-1)^{n^{7}} W_{\left(n^{7}, 2 n^{8}\right)}^{(2)}\right],
$$

which is the proper (anti-)symmetrisation of $\mathscr{A}$.

The D-brane massless spectrum thus comprises the dimensional reduction of tendimensional gauge bosons with gauge group $\operatorname{USp}(8) \otimes \operatorname{SO}(8)$ and fermions in the $(28,1) \oplus$ $(1,36)$ representations. The two gauge group factors (i.e. the branes and the antibranes) are located at different points along the compact $\left(x^{8}, x^{9}\right)$ directions, as depicted in figure 3. This spectrum is obviously Fermi-Bose degenerate, although not supersymmetric. Moreover, in the limit $R, R_{8} \rightarrow \infty$ all massive string levels present Fermi-Bose degeneracy. Actually, in this limit, the closed-string sector and the annulus become supersymmetric, while supersymmetry is broken only by the Möbius amplitude, which reads

$$
\mathscr{M} \simeq \frac{1}{2}\left(N_{\mathrm{D}}-N_{\overline{\mathrm{D}}}\right)\left(V_{8}+S_{8}\right)(-1)^{n^{7}} W_{n^{7}}^{(1)}
$$

This expression describes our choice of distributing branes (antibranes) next to antiorientifolds (orientifolds) planes, thus breaking supersymmetry on their world-volumes. Intuitively, this configuration is as if the fermionic superpartners of the USp(8) and $\mathrm{SO}(8)$ gauge bosons, in the 36 and 28 representations, were exchanged between the two sets of D-branes. 
Note that supersymmetry is restored in the limit $R_{7} \ll \sqrt{\alpha^{\prime}}$, which, in the T-dual description, is equivalent to a large radius limit along the brane world-volume, $\tilde{R}_{7} \equiv$ $\alpha^{\prime} / R_{7} \gg \sqrt{\alpha^{\prime}}$ :

$$
\mathscr{M} \simeq \frac{1}{2}\left(N_{\mathrm{D}}-N_{\overline{\mathrm{D}}}\right)\left[\left(V_{8} \tilde{P}_{2 \tilde{m}_{7}}^{(1)}-S_{8} \tilde{P}_{2 \tilde{m}_{7}+1}^{(1)}\right)-\left(V_{8} \tilde{P}_{2 \tilde{m}_{7}+1}^{(1)}-S_{8} \tilde{P}_{2 \tilde{m}_{7}}^{(1)}\right)\right]
$$

where $\tilde{P}^{(1)}$ denotes the momentum lattice in terms of the dual radius $\tilde{R}_{7}$. This implies that, in this limit, the second term in $(2.6)$ is to be replaced by $c_{1}\left(n_{\mathrm{B}}^{o}-n_{\mathrm{F}}^{o}\right) / \tilde{R}_{7}^{4}$, in analogy with the first term of the closed-string sector, as a consequence of the Scherk-Schwarz supersymmetry breaking. However, since gauge interactions are not localised along this direction, $\tilde{R}_{7}$ cannot become very large, otherwise the gauge theory on the branes would be non perturbative.

\section{The vacuum energy density}

The one-loop vacuum energy receives contributions from the four surfaces of vanishing Euler character

$$
\begin{aligned}
\Lambda(R)= & \frac{1}{2} \int_{\mathscr{F}} \frac{d^{2} \tau}{\tau_{2}^{9 / 2}} \frac{\mathscr{T}(R)}{|\eta|^{10}}+\int_{0}^{\infty} \frac{d \tau_{2}}{\tau_{2}^{9 / 2}} \frac{\mathscr{K}(R)}{\eta^{5}} \\
& +\int_{0}^{\infty} \frac{d \tau_{2}}{\tau_{2}^{9 / 2}} \frac{\mathscr{A}(R)}{\eta^{5}}+\int_{0}^{\infty} \frac{d \tau_{2}}{\tau_{2}^{9 / 2}} \frac{\mathscr{M}(R)}{\hat{\eta}^{5}}
\end{aligned}
$$

where $\mathscr{T}(R), \mathscr{K}(R), \mathscr{A}(R)$ and $\mathscr{M}(R)$ are given in the previous section. Using the Jacobi identity

$$
V_{8}-S_{8}=\frac{1}{2}\left(\frac{\vartheta_{3}^{4}}{\eta^{4}}-\frac{\vartheta_{4}^{4}}{\eta^{4}}-\frac{\vartheta_{2}^{4}}{\eta^{4}}\right) \equiv 0,
$$

and the tadpole condition $N_{\mathrm{D}}=N_{\overline{\mathrm{D}}}=8$, one can cast the various contributions in the simpler form

$$
\begin{gathered}
\mathscr{T} R==\left[\left|\frac{\vartheta_{2}}{\eta}\right|^{8}(-1)^{m} \Gamma_{m, n}^{(1,1)}+\left|\frac{\vartheta_{4}}{\eta}\right|^{8} \Gamma_{m, n+\frac{1}{2}}^{(1,1)}+\left|\frac{\vartheta_{3}}{\eta}\right|^{8}(-1)^{m} \Gamma_{m, n+\frac{1}{2}}^{(1,1)}\right] \Gamma^{(2,2)}(B), \\
\mathscr{K}(R)=\frac{1}{2} \frac{\vartheta_{4}^{4}\left(0 \mid 2 i \tau_{2}\right)}{\eta^{7}\left(2 i \tau_{2}\right)} \sum_{n, n^{7}, n^{8}} e^{-\frac{\pi \tau_{2}}{\alpha^{\prime}}\left[(2 n+1)^{2} R^{2}+\left(2 n^{7} R_{7}\right)^{2}+\left(2 n^{8} R_{8}\right)^{2}\right]}, \\
\mathscr{A}(R)=64 \frac{\vartheta_{4}^{4}\left(0 \mid \frac{i \tau_{2}}{2}\right)}{\eta^{7}\left(\frac{i \tau_{2}}{2}\right)} \sum_{n, n^{7}, n^{8}} e^{-\frac{\pi \tau_{2}}{\alpha^{\prime}}\left[\left(n+\frac{1}{2}\right)^{2} R^{2}+\left(n^{7}+\frac{1}{2}\right)^{2} R_{7}^{2}+\left(n^{8} R_{8}\right)^{2}\right]},
\end{gathered}
$$


and

$$
\mathscr{M}(R)=-8 \frac{\hat{\vartheta}_{2}^{4}\left(0 \mid \frac{1}{2}+\frac{i \tau_{2}}{2}\right)}{\hat{\eta}^{7}\left(\frac{1}{2}+\frac{i \tau_{2}}{2}\right)} \sum_{n, n^{7}, n^{8}} e^{-\frac{\pi \tau_{2}}{\alpha^{\prime}}\left[(2 n R)^{2}+\left(n^{7} R_{7}\right)^{2}+\left(2 n^{8}+1\right)^{2} R_{8}^{2}\right]} .
$$

It is then clear that, for $\tau_{2}>0$, the contributions from the Klein-bottle, annulus and Möbius-strip amplitudes can be made arbitrarily small by taking two of the compact directions, $R$ and $R_{8}$, very large, since

$$
\Lambda(\mathscr{K})+\Lambda(\mathscr{A})+\Lambda(\mathscr{M}) \sim \mathscr{O}\left(e^{-R^{2}} \text { or } e^{-R_{8}^{2}}\right) .
$$

On the other hand, the potentially divergent region $\tau_{2}=0$ is better described, as usual, in terms of the horizontal time $\ell \sim 1 / \tau_{2}$. The amplitudes then read

$$
\begin{aligned}
& \tilde{\mathscr{K}}=\frac{2\left(\alpha^{\prime}\right)^{3 / 2}}{R R_{7} R_{8}} \int_{0}^{\infty} d \ell \frac{\vartheta_{2}^{4}(0 \mid i \ell)}{\eta^{12}(i \ell)} \sum(-1)^{m} e^{-\frac{\pi \ell \alpha^{\prime}}{2}\left[\left(\frac{m}{R}\right)^{2}+\left(\frac{m_{7}}{R_{7}}\right)^{2}+\left(\frac{m_{8}}{R_{8}}\right)^{2}\right],} \\
& \tilde{\mathscr{A}}=\frac{2\left(\alpha^{\prime}\right)^{3 / 2}}{R R_{7} R_{8}} \int_{0}^{\infty} d \ell \frac{\vartheta_{2}^{4}(0 \mid i \ell)}{\eta^{12}(i \ell)} \sum(-1)^{m+m_{7}} e^{-\frac{\pi \ell \alpha^{\prime}}{2}\left[\left(\frac{m}{R}\right)^{2}+\left(\frac{m_{7}}{R_{7}}\right)^{2}+\left(\frac{m_{8}}{R_{8}}\right)^{2}\right]}, \\
& \tilde{\mathscr{M}}=-\frac{4\left(\alpha^{\prime}\right)^{3 / 2}}{R R_{7} R_{8}} \int_{0}^{\infty} d \ell \frac{\hat{\vartheta}_{2}^{4}\left(0 \mid i \ell+\frac{1}{2}\right)}{\eta^{12}\left(i \ell+\frac{1}{2}\right)} \sum(-1)^{m_{8}} e^{-\frac{\pi \ell \alpha^{\prime}}{2}\left[\left(\frac{m}{R}\right)^{2}+\left(\frac{2 m_{7}}{R_{7}}\right)^{2}+\left(\frac{m_{8}}{R_{8}}\right)^{2}\right],}
\end{aligned}
$$

where we have explicitly shown the integration over the transverse-channel modulus $\ell$. In the large radius limit, the integrals are dominated by the closed-string channel infra-red region $\ell \rightarrow \infty$. It is therefore appropriate to rescale the horizontal time as $\alpha^{\prime} \ell / R^{2}$. As a result, the massive string excitations decouple in the large radius limit and the amplitudes reduce to

$$
\begin{aligned}
& \tilde{\mathscr{K}}=\frac{32 R \sqrt{\alpha^{\prime}}}{R_{7} R_{8}} \int_{0}^{\infty} d \ell \sum(-1)^{m} e^{-\frac{\pi \ell}{2}\left[m^{2}+\left(\frac{R}{R_{7}}\right)^{2} m_{7}^{2}+\left(\frac{R}{R_{8}}\right)^{2} m_{8}^{2}\right]}, \\
& \tilde{\mathscr{A}}=\frac{32 R \sqrt{\alpha^{\prime}}}{R_{7} R_{8}} \int_{0}^{\infty} d \ell \sum(-1)^{m+m_{7}} e^{-\frac{\pi \ell}{2}\left[m^{2}+\left(\frac{R}{R_{7}}\right)^{2} m_{7}^{2}+\left(\frac{R}{R_{8}}\right)^{2} m_{8}^{2}\right]}, \\
& \tilde{\mathscr{M}}=-\frac{64 R \sqrt{\alpha^{\prime}}}{R_{7} R_{8}} \int_{0}^{\infty} d \ell \sum(-1)^{m_{8}} e^{-\frac{\pi \ell}{2}\left[m^{2}+4\left(\frac{R}{R_{7}}\right)^{2} m_{7}^{2}+\left(\frac{R}{R_{8}}\right)^{2} m_{8}^{2}\right]} .
\end{aligned}
$$

It is then evident that for $R \sim R_{8} \gg R_{7}$ only $m_{7}=0$ survives, and the contribution of $\tilde{\mathscr{K}}$, $\tilde{\mathscr{A}}$ and $\tilde{\mathscr{M}}$ to the vacuum energy becomes

$$
\begin{aligned}
\tilde{\mathscr{K}}+\tilde{\mathscr{A}}+\tilde{\mathscr{M}} & =\frac{64 R \sqrt{\alpha^{\prime}}}{R_{7} R_{8}} \int_{0}^{\infty} d \ell \sum\left((-1)^{m}-(-1)^{m_{8}}\right) e^{-\frac{\pi \ell}{2}\left[m^{2}+\left(\frac{R}{R_{8}}\right)^{2} m_{8}^{2}\right]} \\
& \simeq \frac{128}{\pi} \frac{R \sqrt{\alpha^{\prime}}}{R_{7} R_{8}} \sum \frac{(-1)^{m}-(-1)^{m_{8}}}{m^{2}+\left(\frac{R}{R_{8}}\right)^{2} m_{8}^{2}},
\end{aligned}
$$


and vanishes in the symmetric case $R=R_{8}$, up to exponentially small corrections, as stated before.

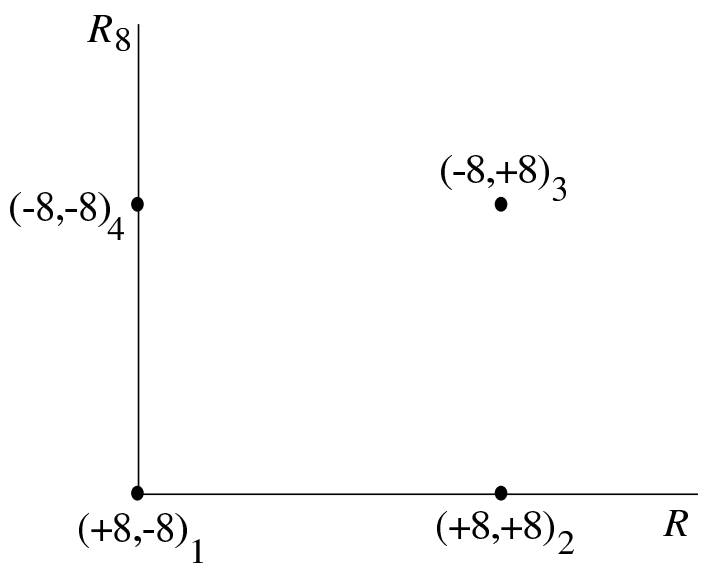

Fig. 4. Mass and charge distribution in the $R, R_{8} \gg R_{7}$ limit.

Actually, these amplitudes can be given a suggestive geometrical interpretation in terms of gravitational and "Maxwell"-type interactions [24], where the condition $R=R_{8}$ would translate into an overall compensation of forces (a BPS-like condition). In the limit $R_{7} \ll R, R_{8}$, we are mainly left with a two-dimensional distribution of charged and massive point-like sources as in figure 4 . Here, the two entries $\left(M_{i}, q_{i}\right)$ in the parenthesis refer to the mass and charge of the $i$-th "particle", where in our conventions a single $07^{+}$plane has both tension and charge equal to -4 while a $\mathrm{D}$ brane carries a (positive) unit of tension and charge. As a result, the overall gravitational force experienced by the system is

$$
\begin{aligned}
F_{\text {Newton }}= & -\left(M_{1} M_{2}+M_{3} M_{4}\right) \log R-\left(M_{1} M_{4}+M_{2} M_{3}\right) \log R_{8} \\
& -\left(M_{1} M_{3}+M_{2} M_{4}\right) \log \sqrt{R^{2}+R_{8}^{2}} \\
= & -128 \log R+128 \log R_{8}+128 \log \sqrt{R^{2}+R_{8}^{2}} .
\end{aligned}
$$

Similarly, the "Maxwell" force due to the R-R field exchange reads

$$
\begin{aligned}
F_{\text {Maxwell }} & =+\left(q_{1} q_{2}+q_{3} q_{4}\right) \log R+\left(q_{1} q_{4}+q_{2} q_{3}\right) \log R_{8}+\left(q_{1} q_{3}+q_{2} q_{4}\right) \log \sqrt{R^{2}+R_{8}^{2}} \\
& =-128 \log R+128 \log R_{8}-128 \log \sqrt{R^{2}+R_{8}^{2}},
\end{aligned}
$$

and thus the total force is

$$
F_{\text {Newton }}+F_{\text {Maxwell }}=-256 \log R+256 \log R_{8} \text {. }
$$


It is then clear that, as stated previously, the condition $R=R_{8}$, required to cancel the leading contribution to the cosmological constant, translates into a (BPS-like) zero-force condition on the distribution of D-branes and $\mathscr{O}$-planes.

The analysis of the torus amplitude is standard. The twisted (odd-winding) sector does not have massless Kaluza-Klein states and thus gives an exponentially suppressed contribution. On the contrary, the untwisted sector contributes with the familiar powerlaw fall-off $1 / R^{7}$. Upon compactification to four dimensions (e.g. on spectator tori) one recovers the expected $1 / R^{4}$ behaviour.

\section{A six-dimensional example with small cosmological constant}

We can now proceed to describe lower-dimensional models with a naturally small oneloop cosmological constant. As before, they consist of suitable combinations of ScherkSchwarz reductions [8] and non-trivial discrete deformations [25].

In general, lower-dimensional orientifolds have a rich structure due to the simultaneous presence of D9 and D5 branes (or T-duals of them), on which Scherk-Schwarz deformations can act differently. However, the proper combination of discrete deformations and modified boundary conditions should guarantee the existence of a non-supersymmetric spectrum with (sets of) branes with Fermi-Bose degeneracy at all massive levels in the large radius limit. As we saw before, this is needed for suppressing the vacuum energy in the gauge (brane) sector.

In six dimensions one could start with the $T^{4} / \mathbb{Z}_{2}$ orbifold model [8]

$$
\begin{aligned}
\mathscr{T}= & \frac{1}{2}\left\{\left(\left|V_{4} O_{4}+O_{4} V_{4}\right|^{2}+\left|S_{4} S_{4}+C_{4} C_{4}\right|^{2}\right) \Gamma_{m, 2 n}\right. \\
& +\left(\left|O_{4} O_{4}+V_{4} V_{4}\right|^{2}+\left|S_{4} C_{4}+C_{4} S_{4}\right|^{2}\right) \Gamma_{m, 2 n+1} \\
& -\left[\left(V_{4} O_{4}+O_{4} V_{4}\right)\left(\bar{S}_{4} \bar{S}_{4}+\bar{C}_{4} \bar{C}_{4}\right)+\text { h.c. }\right] \Gamma_{m+\frac{1}{2}, 2 n} \\
& \left.-\left[\left(O_{4} O_{4}+V_{4} V_{4}\right)\left(\bar{S}_{4} \bar{C}_{4}+\bar{C}_{4} \bar{S}_{4}\right)+\text { h.c. }\right] \Gamma_{m+\frac{1}{2}, 2 n+1}\right\} \Gamma^{(1,1)} \Gamma^{(2,2)}(B) \\
& +\frac{1}{2}\left(\left|V_{4} O_{4}-O_{4} V_{4}\right|^{2}+\left|S_{4} S_{4}-C_{4} C_{4}\right|^{2}\right)\left|\frac{2 \eta}{\vartheta_{2}}\right|^{4} \\
& +\frac{16}{4}\left(\left|O_{4} C_{4}+V_{4} S_{4}-S_{4} O_{4}-C_{4} V_{4}\right|^{2}+\left|O_{4} S_{4}+V_{4} C_{4}-C_{4} O_{4}-S_{4} V_{4}\right|^{2}\right)\left|\frac{\eta}{\vartheta_{4}}\right|^{4} \\
& +\frac{16}{4}\left(\left|O_{4} C_{4}-V_{4} S_{4}-S_{4} O_{4}+C_{4} V_{4}\right|^{2}+\left|O_{4} S_{4}-V_{4} C_{4}-C_{4} O_{4}+S_{4} V_{4}\right|^{2}\right)\left|\frac{\eta}{\vartheta_{3}}\right|^{4},
\end{aligned}
$$


where now the internal lattice has been deformed by the inclusion of a non-trivial $B$-field along a $T^{2}$. As expected, "untwisted" massless fermions (and, in particular, the gravitini) have acquired a mass proportional to the inverse compactification radius. On the contrary, the "twisted" ones stay massless, since they are localised at fixed points and thus do not feel the Scherk-Schwarz deformation. Notice also that the twisted sector is now non chiral as a result of modular invariance.

Following [25], the presence of a discrete $B$-field acts as a projector onto even-winding contributions in $\mathscr{K}$, while no restriction is imposed on the momenta excitations, so that

$$
\begin{aligned}
\mathscr{K}= & \frac{1}{4}\left(V_{4} O_{4}+O_{4} V_{4}-S_{4} S_{4}-C_{4} C_{4}\right)\left(P_{\left(m_{1}, m_{2}, m_{3}, m_{4}\right)}^{(4)}+W_{\left(2 n_{1}, 2 n_{2}, n_{3}, 2 n_{4}\right)}^{(4)}\right) \\
& +\frac{1}{4}\left(O_{4} O_{4}+V_{4} V_{4}-S_{4} C_{4}-C_{4} S_{4}\right) W_{\left(2 n_{1}, 2 n_{2}, n_{3}, 2 n_{4}+1\right)}^{(4)} \\
& +2\left[\left(O_{4} C_{4}+V_{4} S_{4}-S_{4} O_{4}-C_{4} V_{4}\right)+\left(O_{4} S_{4}+V_{4} C_{4}-C_{4} O_{4}-S_{4} V_{4}\right)\right]\left(\frac{\eta}{\vartheta_{4}}\right)^{2}
\end{aligned}
$$

is a proper (anti-)symmetrisation of the torus amplitude. Here, $\left(m_{1}, m_{2}\right)$ and $\left(n_{1}, n_{2}\right)$ denote momenta and windings on the $\Gamma^{(2,2)}(B)$ lattice, while $m_{4}$ and $n_{4}$ refer to the "Scherk-Schwarz" direction. The projected massless spectrum is also non chiral and comprises the graviton, one (unconstrained) two-form and seventeen scalars from the untwisted sector, together with 52 scalars, two (unconstrained) two-forms and eight Dirac fermions from the twisted sector.

The transverse-channel amplitude reveals that while the $\mathscr{O} 9$ plane is a conventional one, the $\mathscr{O} 5$ planes reproduce the geometry described in section 4 (with an additional spectator $S^{1}$ ). Thus, the open string sector involves both D9 branes and D5 brane-antibrane pairs. The corresponding one-loop amplitudes can again be retrieved from [8] and [25], and read

$$
\begin{aligned}
\mathscr{A}_{99}= & \frac{1}{4} \mathbb{I}_{\mathrm{N}}^{2}\left(V_{4} O_{4}+O_{4} V_{4}\right) \sum_{a, b=0, \frac{1}{2}} P_{\left(m_{1}+a, m_{2}+b, m_{3}, m_{4}\right)}^{(4)} \\
& -\frac{1}{4} \mathbb{I}_{\mathrm{N}}^{2}\left(S_{4} S_{4}+C_{4} C_{4}\right) \sum_{a, b=0, \frac{1}{2}} P_{\left(m_{1}+a, m_{2}+b, m_{3}, m_{4}+\frac{1}{2}\right)}^{(4)} \\
& +\frac{1}{4} R_{\mathrm{N}}^{2}\left(V_{4} O_{4}-O_{4} V_{4}\right)\left(\frac{2 \eta}{\vartheta_{2}}\right)^{2}
\end{aligned}
$$

in the D9-D9 sector,

$$
\begin{aligned}
\mathscr{A}_{55}= & \frac{1}{4} \mathbb{I}_{\mathrm{D}}^{2}\left(V_{4} O_{4}+O_{4} V_{4}-S_{4} S_{4}-C_{4} C_{4}\right) W_{\left(n_{1}, n_{2}, n_{3}, n_{4}\right)}^{(1)} \\
& +\frac{1}{4} R_{\mathrm{D}}^{2}\left(V_{4} O_{4}-O_{4} V_{4}+S_{4} S_{4}-C_{4} C_{4}\right)\left(\frac{2 \eta}{\vartheta_{2}}\right)^{2},
\end{aligned}
$$


in the D5-D5 sector,

$$
\begin{aligned}
\mathscr{A}_{\overline{5} \overline{5}}= & \frac{1}{4} \mathbb{I}_{\overline{\mathrm{D}}}^{2}\left(V_{4} O_{4}+O_{4} V_{4}-S_{4} S_{4}-C_{4} C_{4}\right) W_{\left(n_{1}, n_{2}, n_{3}, n_{4}\right)}^{(4)} \\
& +\frac{1}{4} R_{\overline{\mathrm{D}}}^{2}\left(V_{4} O_{4}-O_{4} V_{4}-S_{4} S_{4}+C_{4} C_{4}\right)\left(\frac{2 \eta}{\vartheta_{2}}\right)^{2},
\end{aligned}
$$

in the $\mathrm{D} \overline{5}-\mathrm{D} \overline{5}$ sector, and

$$
\begin{aligned}
\mathscr{A}_{5 \overline{5}}= & \frac{1}{2} \mathbb{I}_{\mathrm{D}} \mathbb{I}_{\overline{\mathrm{D}}}\left(O_{4} O_{4}+V_{4} V_{4}-S_{4} C_{4}-C_{4} S_{4}\right) W_{\left(n_{1}+\frac{1}{2}, n_{2}, n_{3}, n_{4}+\frac{1}{2}\right)}^{(4)}, \\
\mathscr{A}_{95}= & \mathbb{I}_{\mathrm{N}} \mathbb{I}_{\mathrm{D}}\left(O_{4} C_{4}+V_{4} S_{4}-S_{4} O_{4}-C_{4} V_{4}\right)\left(\frac{\eta}{\vartheta_{4}}\right)^{2} \\
& +i R_{\mathrm{N}} R_{\mathrm{D}}\left(O_{4} C_{4}-V_{4} S_{4}-S_{4} O_{4}+C_{4} V_{4}\right)\left(\frac{\eta}{\vartheta_{3}}\right)^{2}, \\
\mathscr{A}_{9 \overline{5}}= & \mathbb{I}_{\mathrm{N}} \mathbb{I}_{\overline{\mathrm{D}}}\left(O_{4} S_{4}+V_{4} C_{4}-C_{4} O_{4}-S_{4} V_{4}\right)\left(\frac{\eta}{\vartheta_{4}}\right)^{2} \\
& +R_{\mathrm{N}} R_{\overline{\mathrm{D}}}\left(O_{4} S_{4}-V_{4} C_{4}-C_{4} O_{4}+S_{4} V_{4}\right)\left(\frac{\eta}{\vartheta_{3}}\right)^{2},
\end{aligned}
$$

in the mixed ones.

Finally, the Möbius-strip amplitudes

$$
\begin{aligned}
\mathscr{M}_{9}= & -\frac{1}{4} \mathbb{I}_{\mathrm{N}}\left[\left(V_{4} O_{4}+O_{4} V_{4}\right) \sum_{a, b=0, \frac{1}{2}}(-1)^{2 a(2 b-1)} P_{\left(m_{1}+a, m_{2}+b, m_{3}, m_{4}\right)}^{(4)}\right. \\
& -\left(S_{4} S_{4}+C_{4} C_{4}\right) \sum_{a, b=0, \frac{1}{2}}(-1)^{2 a(2 b-1)} P_{\left(m_{1}+a, m_{2}+b, m_{3}, m_{4}+\frac{1}{2}\right)}^{(4)} \\
& \left.-\left(V_{4} O_{4}-O_{4} V_{4}\right)\left(\frac{2 \eta}{\vartheta_{2}}\right)^{2}\right] \\
& \mathscr{M}_{5}=-\frac{1}{4} \mathbb{I}_{\mathrm{D}}\left\{\left[V_{4} O_{4}+O_{4} V_{4}+(-1)^{n_{4}}\left(S_{4} S_{4}+C_{4} C_{4}\right)\right]\right. \\
& \times\left(W_{\left(n_{1}, 2 n_{2}+1, n_{3} n_{4}\right)}^{(4)}-(-1)^{n_{1}} W_{\left(n_{1}, 2 n_{2}, n_{3}, n_{4}\right)}^{(4)}\right) \\
& \left.-\left(V_{4} O_{4}-O_{4} V_{4}-S_{4} S_{4}+C_{4} C_{4}\right)\left(\frac{2 \eta}{\vartheta_{2}}\right)^{2}\right\}
\end{aligned}
$$

and

$$
\begin{aligned}
\mathscr{M}_{\overline{5}} & =-\frac{1}{4} \mathbb{I}_{\overline{\mathrm{D}}}\left\{\left[V_{4} O_{4}+O_{4} V_{4}+(-1)^{n_{4}}\left(S_{4} S_{4}+C_{4} C_{4}\right)\right]\right. \\
& \times\left(W_{\left(n_{1}, 2 n_{2}+1, n_{3} n_{4}\right)}^{(4)}+(-1)^{n_{1}} W_{\left(n_{1}, 2 n_{2}, n_{3}, n_{4}\right)}^{(4)}\right) \\
& \left.-\left(V_{4} O_{4}-O_{4} V_{4}+S_{4} S_{4}-C_{4} C_{4}\right)\left(\frac{2 \eta}{\vartheta_{2}}\right)^{2}\right\}
\end{aligned}
$$


which as usual are obtained by the factorisation of $\tilde{\mathscr{K}}$ and $\tilde{\mathscr{A}}$, complete the orientifold projection in the open-string sector, and suggest the following parametrisation of ChanPaton charges

$$
\begin{array}{ll}
\mathbb{I}_{\mathrm{N}}=n+\bar{n}, & R_{\mathrm{N}}=i(n-\bar{n}), \\
\mathbb{I}_{\mathrm{D}}=d_{1}+d_{2}, & R_{\mathrm{D}}=d_{1}-d_{2}, \\
\mathbb{I}_{\overline{\mathrm{D}}}=d+\bar{d}, & R_{\overline{\mathrm{D}}}=i(d-\bar{d}) .
\end{array}
$$

As usual, tadpole conditions fix the overall number of branes

$$
\mathbb{I}_{\mathrm{N}}=16, \quad \mathbb{I}_{\mathrm{D}}=8, \quad \mathbb{I}_{\overline{\mathrm{D}}}=8,
$$

that is again reduced by the presence of the discrete deformation [25].

The massless (anomaly-free) spectrum, obtained by the expansion of $\mathscr{A}$ and $\mathscr{M}$

$$
\begin{aligned}
\mathscr{A}^{(0)} \sim & {\left[n \bar{n}+\frac{1}{2}\left(d_{1}^{2}+d_{2}^{2}\right)+d \bar{d}\right] V_{4} O_{4}+\left[\frac{1}{2}\left(n^{2}+\bar{n}^{2}\right)+d_{1} d_{2}+\frac{1}{2}\left(d^{2}+\bar{d}^{2}\right)\right] O_{4} V_{4} } \\
& -\frac{1}{2}\left(d_{1}^{2}+d_{2}^{2}+d^{2}+\bar{d}^{2}\right) C_{4} C_{4}-\left(d_{1} d_{2}+d \bar{d}\right) S_{4} S_{4} \\
& +2\left(n d_{2}+\bar{n} d_{1}\right)\left(O_{4} C_{4}-S_{4} O_{4}\right)+2(n \bar{d}+\bar{n} d)\left(O_{4} S_{4}-C_{4} O_{4}\right), \\
\mathscr{M}^{(0)} \sim & \frac{1}{2}\left[\left(d_{1}+d_{2}\right) V_{4} O_{4}-(n+\bar{n}+d+\bar{d}) O_{4} V_{4}+\left(d_{1}+d_{2}-d-\bar{d}\right) C_{4} C_{4}\right],
\end{aligned}
$$

comprises a $[\mathrm{U}(8)]_{9} \otimes[\mathrm{USp}(4) \otimes \mathrm{USp}(4)]_{5} \otimes[\mathrm{U}(4)]_{\overline{5}}$ Chan-Paton gauge group with

$$
\begin{aligned}
\text { four scalars : } & (28 \oplus \overline{28} ; 1,1 ; 1) \oplus(1 ; 4,4 ; 1) \oplus(1 ; 1,1 ; 6 \oplus \overline{6}) \\
\text { four scalars : } & (8 ; 1,4 ; 1) \oplus(\overline{8} ; 4,1 ; 1) \oplus(8 ; 1,1 ; \overline{4}) \oplus(\overline{8} ; 1,1 ; 4) \\
\text { one left-handed spinor : } & (1 ; 6,1 ; 1) \oplus(1 ; 1,6 ; 1) \oplus(1 ; 1,1 ; 10 \oplus \overline{10}) \\
& \oplus(8 ; 1,1 ; \overline{4}) \oplus(\overline{8} ; 1,1 ; 4) \\
\text { one right-handed spinor : } & (1 ; 4,4 ; 1) \oplus(1 ; 1,1 ; 16) \oplus(8 ; 1,4 ; 1) \oplus(\overline{8} ; 4,1 ; 1)
\end{aligned}
$$

This spectrum neatly reveals that the mechanism we have described in the previous sections is realised on the D5 system, while the D9 branes feel the familiar Scherk-Schwarz breaking [8]. As a result, in the large radius limit, the one-loop cosmological constant decays like $1 / R^{4}$ in the bulk and on the D9 branes (upon further compactification to four dimensions), while it is exponentially suppressed on the D5 branes due to a Fermi-Bose degenerate spectrum. 


\section{Conclusions and discussions}

We have here presented an explicit example of non-supersymmetric orientifolds with two large transverse dimensions and a naturally small cosmological constant. In the large radius limit, $R=R_{8} \rightarrow \infty$, supersymmetry is restored in the bulk, while the D-brane spectra stay non-supersymmetric, though exhibiting Fermi-Bose degeneracy at all massive string levels. This is the first instance of a vacuum configuration with non-supersymmetric non-abelian gauge sector and a naturally small cosmological constant. In the simplest case, the model contains two "mirror worlds", while the degeneracy is due to an exchange of ordinary superpartners on the two branes.

On the other hand, brane supersymmetry is recovered when another radius $\left(\tilde{R}_{7}\right)$, now longitudinal to the brane world-volume, becomes larger than the string scale. However, $\tilde{R}_{7}$ cannot be made arbitrarily large, since gauge interactions propagate along this direction, and thus, they would become strongly coupled. In the absence of Fermi-Bose degeneracy, this would imply a power-law fall-off of the brane cosmological constant, whose magnitude would be determined, in this case, by the size of this large dimension instead of the string scale.

The main question that remains to be addressed concerns, of course, higher-order corrections. In the following, we give general arguments in favour of their weakness based on the main features of the model presented in this paper. In the large radius limit, $R=R_{8} \rightarrow \infty$, and for fixed world-sheet modulus, the only sizable contribution to $\Lambda$ comes from non-BPS interactions between coincident boundaries (D-branes) and crosscaps $\left(\mathscr{O}_{-}\right.$ planes $)^{\dagger}$. At the one-loop level, such interactions are described by one diagram, the Möbius strip, and the vacuum energy receives two contributions from $\overline{\mathrm{D}}$-branes $/ \mathscr{O}^{+}$-plane and Dbranes $/ \overline{\mathscr{O}}^{-}$-plane interactions. Since the projection of the two orientifold planes, which locally breaks supersymmetry, is opposite in the two "mirror worlds", the two contributions to the vacuum energy, equal in magnitude, have opposite signs, thus yielding a vanishing result.

A similar mechanism is therefore expected to take place in higher-genus surfaces containing zero or one crosscap, while a problem might emerge for surfaces containing two

$\dagger$ In fact, the non-supersymmetric (i.e. non-vanishing) brane/brane and $\mathscr{O}$-plane/OOO-plane interactions are exponentially suppressed in $R$ and $R_{8}$. 
crosscaps, together with any boundary. $\ddagger$ The simplest surface of this type arises at genus $3 / 2$, and has one hole and two crosscaps. Again, one has to sum over the two contributions associated to the two different sets of "massless" brane/OO-plane configurations. A preliminary analysis of such a diagram indicates indeed that the two contributions are equal but now come with even sign, yielding a brane vacuum energy of the order of $M_{s}^{4}$. However, in the limit $\tilde{R}_{7} \gg \ell_{s}$, this contribution is expected to vanish, since supersymmetry is recovered. Hence, one is left with potential contributions from various degeneration limits of the world-sheet modular parameters (period matrix).

To better elucidate this point, let us summarise here the one-loop case studied in detail in sections 4 and 5 . In the ultraviolet limit, $\tau_{2} \rightarrow 0$, that is not in the fundamental domain for the modulus of the torus, the (transverse-channel) Klein-bottle, annulus and Möbius amplitudes contribute as

$$
\begin{aligned}
\tilde{\mathscr{K}}+\tilde{\mathscr{A}}+\tilde{\mathscr{M}} & \simeq \frac{64 \tilde{R}_{7}}{R R_{8}} \sum_{m, m_{8}}\left[\left(1+\frac{N_{\mathrm{D}} N_{\overline{\mathrm{D}}}}{64}\right)(-1)^{m}-\frac{N_{\mathrm{D}}+N_{\overline{\mathrm{D}}}}{8}(-1)^{m_{8}}\right] \int_{0}^{\infty} d \ell e^{-\frac{\pi \ell}{2}\left(\frac{m^{2}}{R^{2}}+\frac{m_{8}^{2}}{R_{8}^{2}}\right)} \\
& =\frac{64 \tilde{R}_{7}}{R^{2}}\left(1-\frac{N_{\mathrm{D}}}{8}\right)\left(1-\frac{N_{\overline{\mathrm{D}}}}{8}\right) \sum_{m, m_{8}}(-1)^{m} \int_{0}^{\infty} d \ell e^{-\frac{\pi \ell}{2} \frac{m^{2}+m_{8}^{2}}{R^{2}}},
\end{aligned}
$$

where, for convenience, we have restored the Chan-Paton factors for branes $\left(N_{\mathrm{D}}\right)$ and antibranes $\left(N_{\overline{\mathrm{D}}}\right)$, located next to the $\overline{\mathscr{O}}^{-}$and $\mathscr{O}^{+}$planes, respectively. Clearly, the ultraviolet contribution is due to the sum of local tadpoles of all massless string states, and vanishes if such tadpoles are absent, i.e. when $N_{\mathrm{D}}=N_{\overline{\mathrm{D}}}=8$.

On the other hand, the infrared region, $\tau_{2} \rightarrow \infty$, may receive contributions only from zero-winding sectors. The large radius behaviour is now determined by the restoration of supersymmetry. In the closed-string sector, one finds the familiar contribution $\left(n_{B}^{c}-\right.$ $\left.n_{F}^{c}\right) / R^{4}$ which is suppressed below the experimental limit for extra dimensions of submillimetre size. In the open-string sector, however, one gets a term proportional to $\left(n_{B}^{o}-\right.$ $\left.n_{F}^{o}\right) / \tilde{R}_{7}^{4}$, which can not be sufficiently dumped since $\tilde{R}_{7}^{-1}$ is in the $\mathrm{TeV}$ region. More precisely, following our general analysis in section 4, the Möbius amplitude contributes with

$$
\mathscr{M} \simeq 4\left(N_{\mathrm{D}}-N_{\overline{\mathrm{D}}}\right) \int_{0}^{\infty} \frac{d t}{t^{3}} \sum_{n^{7}} e^{-\frac{\pi}{2 t}\left(2 n^{7}+1\right)^{2} \tilde{R}_{7}^{2}} \sim \frac{N_{\mathrm{D}}-N_{\overline{\mathrm{D}}}}{\tilde{R}_{7}^{4}}
$$

$\ddagger$ Indeed, a general classification of non-oriented and topologically inequivalent Riemann surfaces implies that there are only three classes of diagrams characterised by 0,1 or 2 crosscaps and an arbitrary number of holes and handles. 
However, because of Fermi-Bose degeneracy in the D-brane spectrum $\left(N_{\mathrm{D}}=N_{\overline{\mathrm{D}}}=8\right)$, this term is absent and the next correction is exponentially suppressed in $\tilde{R}_{7} \gg \ell_{s}$.

Note the similarity of these models with the heterotic duals of Fermi-Bose degenerate closed-string vacua of ref. [13], where the inverse of the heterotic radius is proportional to the type IIA string coupling constant, and supersymmetry is restored in the large radius limit. This similarity, together with the property of local tadpole cancellation in the vacuum energy, suggests that higher order corrections could be suppressed, at least in a class of models satisfying additional constraints, along the lines of the closed-string examples [26]. In any case, the precise analysis of higher-genus corrections and possible extra conditions in our constructions remains an interesting open problem.

Acknowledgement We thank Tom Taylor for enlightening discussions. C.A. would like to acknowledge the Physics Department of Oxford University for hospitality during the completion of this work. This work was supported in part by the European Commission under the RTN contract HPRN-CT-2000-00148, the INTAS contract 55-1-590 and the Royal Society. 


\section{References}

[1] I. Antoniadis, "A Possible New Dimension at a Few TeV," Phys. Lett. B246 (1990) 377.

[2] N. Arkani-Hamed, S. Dimopoulos and G. Dvali, "The Hierarchy Problem and New Dimensions at a Millimetre," Phys. Lett. B429 (1998) 263, arXiv:hep-ph/9803315 and "Phenomenology, astrophysics and cosmology of theories with sub-millimetre dimensions and TeV scale quantum gravity," Phys. Rev. D59 (1999) 086004 arXiv:hepph/9807344]; I. Antoniadis, N. Arkani-Hamed, S. Dimopoulos and G. R. Dvali, "New dimensions at a millimetre to a Fermi and superstrings at a TeV," Phys. Lett. B436 (1998) 257 arXiv:hep-ph/9804398].

[3] I. Antoniadis and C. Bachas, "Branes and the gauge hierarchy," Phys. Lett. B450 (1999) 83 arXiv:hep-th/9812093; N. Arkani-Hamed, S. Dimopoulos and J. MarchRussell, "Stabilisation of sub-millimetre dimensions: The new guise of the hierarchy problem," Phys. Rev. D63 (2001) 064020 [arXiv:hep-th/9809124].

[4] I. Antoniadis, K. Benakli, A. Laugier and T. Maillard, "Brane to bulk supersymmetry breaking and radion force at micron distances," Nucl. Phys. B662 (2003) 40 arXiv:hep$\mathrm{ph} / 0211409]$.

[5] C. P. Burgess, R. C. Myers and F. Quevedo, "A naturally small cosmological constant on the brane?," Phys. Lett. B495 (2000) 384 [arXiv:hep-th/9911164]; Y. Aghababaie, C. P. Burgess, S. L. Parameswaran and F. Quevedo, "Toward a naturally small cosmological constant from branes in 6D supergravity," arXiv:hep-th/0304256.

[6] J. Scherk and J. H. Schwarz, "How To Get Masses From Extra Dimensions," Nucl. Phys. B153 (1979) 61 and "Spontaneous Breaking Of Supersymmetry Through Dimensional Reduction," Phys. Lett. B82 (1979) 60.

[7] R. Rohm, "Spontaneous Supersymmetry Breaking In Supersymmetric String Theories," Nucl. Phys. B237 (1984) 553; C. Kounnas and M. Porrati, "Spontaneous Supersymmetry Breaking In String Theory," Nucl. Phys. B310 (1988) 355; S. Ferrara, C. Kounnas, M. Porrati and F. Zwirner, "Superstrings With Spontaneously Broken Supersymmetry And Their Effective Theories," Nucl. Phys. B318 (1989) 75.

[8] I. Antoniadis, E. Dudas and A. Sagnotti, "Supersymmetry breaking, open strings and M-theory," Nucl. Phys. B544 (1999) 469 [arXiv:hep-th/9807011]; I. Antoniadis, G. D'Appollonio, E. Dudas and A. Sagnotti, "Partial breaking of supersymmetry, open strings and M-theory," Nucl. Phys. B553 (1999) 133 [arXiv:hep-th/9812118].

[9] A. L. Cotrone, "A $\mathbb{Z}_{2} \times \mathbb{Z}_{2}$ orientifold with spontaneously broken supersymmetry," Mod. Phys. Lett. A 14 (1999) 2487 [arXiv:hep-th/9909116].

[10] H. Itoyama and T. R. Taylor, "Supersymmetry Restoration In The Compactified O(16) X O(16)-Prime Heterotic String Theory," Phys. Lett. B186 (1987) 129.

[11] I. Antoniadis, S. Dimopoulos and G. R. Dvali, "Millimetre range forces in superstring theories with weak-scale compactification,"Nucl. Phys. B516 (1998) 70 arXiv:hepph/9710204. 
[12] S. Kachru, J. Kumar and E. Silverstein, "Vacuum energy cancellation in a nonsupersymmetric string," Phys. Rev. D59 (1999) 106004 arXiv:hep-th/9807076.

[13] J. A. Harvey, "String duality and non-supersymmetric strings," Phys. Rev. D59 (1999) 026002 arXiv:hep-th/9807213.

[14] S. Kachru and E. Silverstein, "On vanishing two loop cosmological constants in nonsupersymmetric strings," JHEP 9901 (1999) 004 arXiv:hep-th/9810129; R. Iengo and C. J. Zhu, "Evidence for non-vanishing cosmological constant in non-SUSY superstring models," JHEP 0004 (2000) 028 arXiv:hep-th/9912074.

[15] R. Blumenhagen and L. Görlich, "Orientifolds of non-supersymmetric, asymmetric orbifolds," Nucl. Phys. B551 (1999) 601 arXiv:hep-th/9812158.

[16] C. Angelantonj, I. Antoniadis and K. Forger, "Non-supersymmetric type I strings with zero vacuum energy," Nucl. Phys. B555 (1999) 116 arXiv:hep-th/9904092].

[17] S. Sugimoto, "Anomaly cancellations in type I D9-D9-bar system and the USp(32) string theory," Prog. Theor. Phys. 102 (1999) 685 [arXiv:hep-th/9905159; I. Antoniadis, E. Dudas and A. Sagnotti, "Brane supersymmetry breaking," Phys. Lett. B464 (1999) 38 [arXiv:hep-th/9908023]; G. Aldazabal and A. M. Uranga, "Tachyon-free non-supersymmetric type IIB orientifolds via brane-antibrane systems," JHEP 9910 (1999) 024 arXiv:hep-th/9908072.

[18] C. Angelantonj and A. Sagnotti, "Open strings," Phys. Rept. 371 (2002) 1 [Erratumibid. 376 (2003) 339] arXiv:hep-th/0204089.

[19] A. Sagnotti, "Open Strings And Their Symmetry Groups," Cargese Summer Institute on Non-Perturbative Methods in Field Theory, Cargese, France, 1987 arXiv:hepth/0208020]; M. Bianchi and A. Sagnotti, "On The Systematics Of Open String Theories," Phys. Lett. B247 (1990) 517 and "Twist Symmetry And Open String Wilson Lines," Nucl. Phys. B361 (1991) 519; P. Horava, "Strings On World Sheet Orbifolds," Nucl. Phys. B327 (1989) 461.

[20] E. Dudas, "Theory and phenomenology of type I strings and M-theory," Class. Quant. Grav. 17 (2000) R41 arXiv:hep-ph/0006190.

[21] E. Witten, "Toroidal compactification without vector structure," JHEP 9802 (1998) 006 arXiv:hep-th/9712028.

[22] M. Borunda, M. Serone and M. Trapletti, "On the quantum stability of type IIB orbifolds and orientifolds with Scherk-Schwarz SUSY breaking," Nucl. Phys. B 653 (2003) 85 arXiv:hep-th/0210075.

[23] M. Bianchi, G. Pradisi and A. Sagnotti, "Toroidal compactification and symmetry breaking in open string theories," Nucl. Phys. B376 (1992) 365.

[24] J. Polchinski, "Dirichlet-Branes and Ramond-Ramond Charges," Phys. Rev. Lett. 75 (1995) 4724 arXiv:hep-th/9510017.

[25] C. Angelantonj, "Comments on open-string orbifolds with a non-vanishing $B_{a b}$," Nucl. Phys. B566 (2000) 126 [arXiv:hep-th/9908064.

[26] E. Silverstein, private communication. 